\title{
RETRACTED ARTICLE: Piezonuclear evidences from tensile and compression tests on steel
}

\author{
Stefano Invernizzi - Oscar Borla • \\ Giuseppe Lacidogna $\cdot$ Alberto Carpinteri
}

Received: 21 November 2013/Accepted: 8 May 2014/Published online: 26 June 2014

(C) The Author(s) 2014

This article has been withdrawn by the Publisher and the Society in agreement with the Editor-in-Chief due to conflict of interest reasons. In a commitment to scientific integrity we decided to withdraw the article as the editorial process had been compromised.

S. Invernizzi $(\bowtie) \cdot$ O. Borla $\cdot$ G. Lacidogna

A. Carpinteri

Department of Structural, Geotechnical and Building

Engineering, Politecnico di Torino, Corso Duca degli

Abruzzi 24, 10129 Turin, Italy

e-mail: stefano.invernizzi@polito.it 